\title{
Frequência e repercussão da sobrecarga de cuidadoras familiares de idosos com demência
}

\author{
Frequency and impact of the burden on family caregivers of elderly with dementia
}

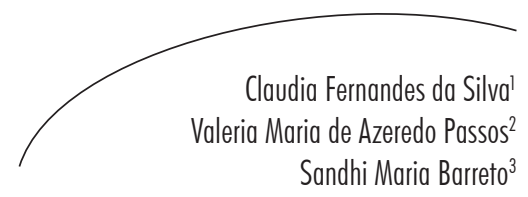

Resumo

Objetivos: Investigar a prevalência e os fatores associados a sobrecarga, transtornos mentais comuns e autopercepção da memória das cuidadoras familiares de idosos com demência. Métodos: Estudo transversal e analítico, com amostra de conveniência de 58 cuidadoras familiares que participaram dos grupos de apoio à família da pessoa com demência. Instrumentos utilizados: questionário sociodemográfico, de hábitos de vida, morbidade referida, uso de medicamentos e de serviços de saúde, Escala Zarit Burden Interview, Self Report Questionnaire 20 (SRQ -20), Prospective and Retrospective Memory Questionnaire (PRMQ-10) e Escala Funcional de Pfeffer. Resultados: Predominaram as seguintes características: 42 eram filhas $(72,4 \%)$, adultas $(63,8 \%)$, com idade inferior a 60 anos $(63,8 \%)$ e grau de instrução superior completo $(46,6 \%)$. Dentre os problemas de saúde autorreferidos, os mais prevalentes foram dores nas costas $(63,8 \%)$, problemas articulares $(60,3 \%)$, colesterol alto $(51,7 \%)$ e hipertensão arterial $(44,8 \%)$. Os medicamentos mais utilizados foram os anti-hipertensivos $(38,9 \%)$ e antidepressivos $(31,5 \%)$. Os dados evidenciaram que o grau de sobrecarga foi de leve a moderado $(51,7 \%)$. No modelo final, observou-se associação da sobrecarga com ocupação ( $p=0,014)$, prática de esporte $(p=0,010)$, ter religião $(p=0,032)$ e tempo na função de cuidadora $(p=0,046)$. Com relação aos transtornos mentais menores, as associações relevantes foram prática de esporte $(p=0,028)$, dores nas costas $(p=0,026)$ e tempo de cuidado diário $(p=0,037)$. As queixas de memória foram associadas estatisticamente ao uso de antidepressivo ( $p=0,034)$. Conclusões: Apesar das informações e orientações sobre a atividade de cuidar nos grupos de apoio, as cuidadoras ainda apresentaram sobrecarga, reforçando a necessidade de um trabalho preventivo, de orientação e tratamento.

1 Programa de Pós-graduação em Ciências Aplicadas à Saúde do Adulto. Universidade Federal de Minas Gerais. Belo Horizonte, MG, Brasil.

2 Departamento de Clínica Médica, Faculdade de Medicina. Universidade Federal de Minas Gerais. Belo Horizonte, MG, Brasil.

3 Departamento de Medicina Preventiva e Social, Faculdade de Medicina. Universidade Federal de Minas Gerais. Belo Horizonte, MG, Brasil.

Correspondência / Correspondence

Claudia Fernandes da Silva

E-mail: claudiafono@terra.com.br

\section{Palavras-chave:}

Esgotamento profissional. Cuidadores. Doença de Alzheimer. Transtornos mentais. Depressão. Ansiedade. 


\section{Abstract}

Objectives: To investigate the prevalence and factors associated to burden, common mental distress and memory self-perception of family caregivers of elderly with dementia. Methods: A cross-sectional and analytical study with a convenience sample of 58 family caregivers who participated in support groups for families of people with dementia. Instruments used: socio-demographic questionnaire, lifestyle habits, referred morbidity, use of medication and health services, Zarit Burden Interview Scale, Self Report Questionnaire SRQ-20, Prospective and Retrospective Memory Questionnaire (PRMQ-10) and Pfeffer Functional Scale. Results: From the 58 caregivers interviewed, the following characteristics predominated: 42 were daughters $(72.4 \%)$, adults $(63.8 \%)$, ages below 60 years old $(63.8 \%)$, with complete college degree (46.6\%). Among the selfreported health problems, the most prevalent were the presence of back $(63.8 \%)$ and joints pains (60.3\%), high cholesterol (51.7\%) and hypertension (44.8\%). The most used drugs were: anti-hypertensive (38.9\%) and antidepressant (31.5\%). Data showed that the degree of burden was from mild to moderate (51.7\%). The final model showed an association of burden with occupation $(p=0,014)$, sports $(p=0,010)$, religion $(p=0,032)$ and time exercising the caregiver activities $(p=0,046)$. Related to the minor mental disorders the relevant associations were sports $(p=0,028)$, back pain $(p=0,026)$ and daily care time $(p=0,037)$. Memory complaints were statistically associated to the use of antidepressants $(p=0,034)$. Conclusions: Despite the information and guidance given to the caregivers about caregiver activities in the support groups, they showed an overload of burden, reinforcing the need of preventive work, guidance and treatment.
Key words: Professional Burnout. Caregivers. Alzheimer's Disease. Mental Disorders. Depression. Anxiety.

\section{INTRODUÇÃO}

Os idosos constituem o grupo etário que mais cresce no Brasil. O país possui cerca de 19 milhões de pessoas com 60 anos ou mais, o que representa mais de $10 \%$ da população brasileira. Estimativas nacionais indicam que esse contingente atingirá 32 milhões em 2025 e fará do país o sexto em número de idosos no mundo. ${ }^{1} \mathrm{O}$ envelhecimento é uma conquista da humanidade, pois possibilita a maior convivência dos idosos com seus familiares. No entanto, ele também pode vir acompanhado de incapacidade e problemas de saúde, tais como algumas doenças crônicas e degenerativas, entre elas as demências. ${ }^{2}$

No Brasil, os idosos são responsáveis pelo maior percentual de gastos públicos com saúde. ${ }^{3}$ O modelo hospitalar contribuiu para que os cuidados no domić́lio não fossem incentivados. Nos últimos anos, essa prática ressurgiu devido à rápida transição demográfica e epidemiológica e à elevação dos custos assistenciais à saúde dos idosos. ${ }^{4}$ Em muitos países e no Brasil, a tendência atual, devido à redução de custos da assistência hospitalar e institucional aos idosos incapacitados, é de indicar a permanência desses idosos em suas casas, sob os cuidados de suas famílias, visando também ao conforto e dignidade. ${ }^{5}$

Diante dessa nova demanda, surge o cuidador familiar, geralmente o parceiro, filho ou outro familiar próximo. Essas são pessoas que, de uma hora para outra, são obrigadas a lidar com familiares fragilizados física e cognitivamente. Os cuidadores se deparam com tarefas múltiplas e desafiadoras, que incluem aceitar o diagnóstico, administrar o conflito familiar, reprogramar o futuro e, principalmente, na realização das atividades diárias, administrar os medicamentos e o suporte financeiro e legal ao paciente. Com isso, o cuidador ficará vulnerável a doenças físicas, depressão, perda de peso, insônia, ao uso de álcool e de medicamentos psicotrópicos. ${ }^{6}$

Sentimentos de depressão e ansiedade ocorrem quando o cuidador percebe que sua estrutura familiar está abalada devido à modificação dos papéis sociais. Ele é constantemente testado em sua "capacidade de discernimento e adaptação à nova realidade, que exige, além da dedicação, responsabilidade, paciência e abnegação". ${ }^{2}$ 
Estima-se que entre $60 \%$ e $70 \%$ dos cuidadores familiares de pacientes com demência têm estresse e problemas médicos ou psiquiátricos. Muitos desses cuidadores apresentam maior probabilidade de ter ansiedade, depressão e um sentimento de isolamento, assim como aumento da taxa de morbidade e mortalidade.?

O ato de cuidar é silencioso e solitário, sem uma garantia de retribuição, de reconhecimento e de afeto, podendo gerar sentimentos ambivalentes do cuidador em relação ao idoso, como culpa, raiva, medo e angústia. Por outro lado, o ato de cuidar pode gerar sentimentos de amor, gratidão e carinho, como forma de manifestar o reconhecimento pelos cuidados e atenção recebidos em outro momento da vida, tornando paciente e cuidador cúmplices nessa nova relação. ${ }^{8}$

À medida que a doença evolui, a demanda de cuidados com o idoso também aumenta, gerando um aumento das dificuldades e, consequentemente, da sobrecarga. $\mathrm{O}$ nível de sobrecarga de trabalho do cuidador, assim como de estresse e funcionamento cognitivo, também está intimamente relacionado ao grau de dependência do paciente. Quanto mais dependente e comprometido cognitivamente for o paciente, maior será sua necessidade de cuidado, o que faz com que o cuidador tenha menos tempo livre para si mesmo, aumentando sua ansiedade em relação à função de cuidar.'

A pessoa que cuida ininterruptamente precisa, além do esforço físico, estar atenta à execução de determinados procedimentos que exigem concentração e planejamento antecipado do que será feito, visando obter os resultados satisfatórios no cuidado que tem que ser realizado. ${ }^{8}$

Além dos problemas emocionais, a memória do cuidador também pode ser afetada. Podemos considerar a memória como uma agenda interna que organiza os eventos ocorridos no passado e registra os planos para o futuro. As pessoas planejam o seu dia a dia na intenção de que todas as tarefas e atividades de lazer sejam realizadas no momento certo. A ocorrência de falhas nesse planejamento cotidiano é possível. ${ }^{10}$
O indivíduo que apresenta algum prejuízo da memória, mesmo que não patológico, pode se queixar da memória com maior frequência. ${ }^{11}$ Supõe-se que o estresse é um fator emocional, cuja predominância em certos períodos da vida do adulto jovem pode elevar o autorrelato de falhas de memória. Entre os idosos, as queixas de memória prospectiva e retrospectiva podem ser mais relatadas devido à presença de sintomas depressivos. ${ }^{10}$

O presente estudo tem como objetivo avaliar se cuidar de pacientes familiares com demência aumenta a sobrecarga dos cuidadores, e se isso pode estar associado ao aumento de transtornos mentais comuns e de queixas subjetivas de memória. O estudo também visa identificar as variáveis que podem comprometer a manutenção do autocuidado e da qualidade de vida dos cuidadores, buscando a preservação de um atendimento mais adequado ao idoso incapacitado.

\section{METODOLOGIA}

\section{Delineamento}

Estudo transversal e analítico, com participação de cuidadores familiares voluntários, realizado de março a setembro de 2010.

\section{População do estudo}

Amostra de conveniência de 65 cuidadores familiares que participaram de reuniões mensais dos grupos de apoio à família da pessoa com demência em Belo Horizonte: Associação Brasileira de Alzheimer (ABRAz), Hospital do IPSEMG, Igreja São Mateus e Centro Jenny Faria de Assistência à Saúde do Idoso e da Mulher da Universidade Federal de Minas Gerais (UFMG).

\section{Procedimentos}

Definiu-se cuidador como um familiar próximo, responsável direto pelos cuidados do idoso, que não recebe remuneração e que cuida do paciente há pelo menos três meses, por no 
mínimo quatro horas por dia e pelo menos três vezes por semana. ${ }^{2,12,13}$

Para participar da pesquisa, o cuidador deveria ser maior de 18 anos e ter capacidade física e mental para responder ao questionário. Apenas um cuidador foi excluído, por apresentar provável transtorno psiquiátrico. Aqueles que aceitaram participar da pesquisa assinaram o termo de consentimento livre e esclarecido e responderam à entrevista. As pessoas que se recusaram a participar do estudo preencheram o formulário de perdas com registro de alguns dados sociodemográficos. Algumas pessoas se recusaram a participar do estudo e também a preencher o formulário de perdas. A aplicação da entrevista se deu de forma individual, pela pesquisadora deste estudo (CFS), na residência do cuidador, com cerca de uma hora de duração.

A avaliação da sobrecarga foi feita com a utilização da escala Zarit Burden Interview, ${ }^{14}$ validada no Brasil, composta por 22 questões que avaliam o quanto as atividades do cuidado têm impacto sobre a vida social, o bem-estar físico e emocional e as finanças do cuidador, pontuadas de 0 a 88, apresentando os seguintes pontos de corte: 0-21,ausência de sobrecarga; 21-40, sobrecarga leve a moderada; 41-60, sobrecarga moderada a severa; e 61-88, sobrecarga severa.

Para avaliação dos transtornos mentais comuns, utilizou-se o Self Report Questionnaire 20 (SRQ), ${ }^{15,16}$ questionário composto por 20 questões relacionadas a transtornos não-psicóticos, tendo como pontos de corte 7 para mulheres e 6 para homens, para a caracterização do sofrimento mental.

As queixas de memória foram avaliadas por meio do questionário Prospective and Retrospective Memory Questionnaire (PRMQ-10), ${ }^{10}$ na versão em português, sendo uma medida de autoavaliação e não uma medida direta de memória. Dos dez itens, cinco referem-se à memória prospectiva e cinco, à memória retrospectiva. Cada pergunta é respondida como escala Likert de frequência e intensidade. $\mathrm{O}$ escore máximo é 50 pontos e reflete um alto índice de queixas de memória, enquanto o escore mínimo é 10 pontos para um baixo índice de queixas de memória. O ponto de corte para um baixo ou alto índice de queixas é igual a 20 pontos.

As variáveis sociodemográficas (idade, escolaridade, estado civil, ocupação), de hábitos de vida (fumo, álcool, atividade física, lazer e sono), morbidade referida e uso de medicamentos e de serviços de saúde foram baseadas no protocolo ELSA. ${ }^{17}$ As variáveis que descrevem a tarefa de cuidar foram: grau de parentesco com o paciente, tempo e número de horas do dia despendidas na função de cuidador, e tempo de diagnóstico da demência. ${ }^{13}$ Para a avaliação do grau de dependência do idoso a ser cuidado, utilizou-se a Escala Funcional de Pfeffer, ${ }^{18}$ composta por dez itens, que evidencia a funcionalidade do idoso com demência pelo grau de independência para a realização das atividades instrumentais de vida diária. Seu escore mínimo é 0 e o máximo é 30 .

\section{Análise Estatística}

Os dados foram inseridos em um banco de dados no software Epi-Info, versão original em português, atualizada para a 3.3.2 (de fev/2005), a partir das variáveis definidas. A análise estatística foi realizada por meio do software estatístico STATA, versão 10.0.

Por se tratar de amostra de conveniência e pelo fato de os testes terem sido aplicados somente uma vez, a estimativa da confiabilidade foi medida por meio da consistência interna das escalas utilizadas nesta pesquisa, pelo coeficiente Alpha de Cronbach.

Foi feita análise exploratória dos dados para se obter as características da população estudada e a prevalência da sobrecarga dos cuidadores, dos transtornos mentais comuns e das queixas de memória. A investigação dos fatores associados à sobrecarga, transtornos mentais comuns e queixas de memória foi realizada com o uso da análise de tabelas de contingência, testando as associações entre as variáveis por meio dos testes Qui-quadrado e do teste exato de Fisher. Após a análise bivariada, foram feitos modelos finais, 
utilizando a análise de regressão logística múltipla para determinar a força de associação entre as variáveis, visando à estimativa do risco (odds ratio) ajustada pelas variáveis contidas nesse modelo final. Para todos os testes usados, considerou-se um nível de significância de $p<0,05$.

O projeto foi aprovado pelo Comitê de Ética em Pesquisa da Universidade Federal de Minas Gerais (n.0306.0.203.000-10). Foi obtido o consentimento informado de cada cuidador.

\section{RESULTADOS}

Em um universo de 119 participantes dos grupos de apoio, 55 cuidadores eram da Igreja São Mateus, 29 do Hospital do IPSEMG, 19 do Centro Jenny Faria de Assistência à Saúde do Idoso e da Mulher-UFMG e 16 da Associação Brasileira de Alzheimer (ABRAZ). Responderam às entrevistas 65 pessoas (54,6\%), 28 (23,5\%) preencheram o formulário de perdas, e 26 $(21,8 \%)$ recusaram qualquer participação.

A comparação entre os participantes do estudo e aqueles que preencheram o formulário de perdas não revelou diferenças significativas para sexo $\left(x^{2}=0,23 p=0,63\right)$, idade $\left(x^{2}=4,16\right.$ $p=0,12)$, situação conjugal $\left(x^{2}=1,78 p=0,18\right)$, situação profissional $\left(x^{2}=0,37 p=0,54\right)$, ou grau de instrução $\left(x^{2}=2,25 p=0,32\right)$. Entretanto, percebeu-se maior percentual de pessoas ativas, $20(71,4 \%)$, do que de aposentadas, $8(28,6 \%)$, que não quiseram participar do estudo $\left(x^{2}=\right.$ $3,91 p=0,04)$.

Dos 65 entrevistados, 58 eram do sexo feminino. Dada a grande representação feminina da amostra, os homens foram excluídos desta análise, devido às diferenças entre os gêneros no que se refere ao estresse do cuidador.

Das 58 cuidadoras incluídas no estudo, 42 $(72,4 \%)$ eram filhas e adultas $(63,8 \%)$ com menos de 60 anos. A idade variou de 30 a 88 anos (média $=58,2 \mathrm{e} \mathrm{dp}=10,4)$. Grande parte das cuidadoras tinha grau de instrução superior completo $(46,6 \%)$ e trabalhava $(53,5 \%)$, predominando as profissões de professora e secretária. Apenas sete
$(10,77 \%)$ tinham ocupação exclusiva no lar. Em relação à tarefa de cuidar, $32(55,2 \%)$ exerciam a função de cuidadora há menos de 60 meses. Não houve diferença em relação ao tempo de cuidado diário ( $50 \%$ ou durante todo o dia), mas observou-se que a maioria, 35 (60,3\%), não era cuidador único, e que os familiares cuidados tiveram o diagnóstico de demência há mais de 60 meses $(50 \%)$.

Quanto à saúde e hábitos de vida, 46 (79,3\%) cuidadoras relataram atividades de lazer e 31 $(53,5 \%)$ a prática de esportes. Vinte e nove $(50 \%)$ faziam uso de bebida alcoólica e apenas três $(5,1 \%)$ fumavam. A maioria, 36 (66,1\%), dormia menos de oito horas por noite.

Entre as doenças autorreferidas, as mais prevalentes foram a presença de dores nas costas ou problemas de coluna $(63,8 \%)$, dores articulares (60,3\%), nível sérico aumentado de colesterol (51,7\%), hipertensão arterial (44,8\%), anemia (31\%) e asma/bronquite (13,8\%). Das 58 cuidadoras, $53(91,3 \%)$ relataram ter tomado alguma medicação nos últimos sete dias, indicada por consulta médica $(87,9 \%)$. Os medicamentos mais utilizados foram os anti-hipertensivos $(38,9 \%)$, antidepressivos (31,5\%) e hormônio tireoidiano $(20,4 \%)$. Trinta e quatro $(58,6 \%)$ utilizaram o plano de saúde e fizeram consultas médicas nos últimos 12 meses (94,8\%). Em relação à espiritualidade, $50(86,2 \%)$ cuidadoras relataram ter religião e $47(81,0 \%)$ frequentavam atividades religiosas.

Observou-se neste estudo uma boa consistência interna das respostas às escalas de sobrecarga (Zarit $\alpha=0,72$ ), transtornos mentais comuns (SRQ $\alpha=0,62$ ), queixas subjetivas de memória (PRMQ-10 $\alpha=0,74)$ e da avaliação funcional do idoso com demência (escala de Pfeffer $\alpha=0,88)$.

Não foi observada associação significativa entre a avaliação funcional e as variáveis dependentes. Do total de respostas dadas pelos participantes da pesquisa no questionário de Pfeffer, 80,3\% responderam a categoria 3 (totalmente dependente) para todas as perguntas. 
Pouco mais da metade das cuidadoras apresentou grau de sobrecarga de leve a moderado ( $\mathrm{n}=30 ; 51,7 \%)$, seguido de moderado a severo ( $\mathrm{n}=28 ; 48,3 \%)$. Não houve relato de sobrecarga leve e severa nessa população. Dessa forma, foi feita a comparação entre cuidadoras que apresentaram sobrecarga leve e aquelas que apresentaram sobrecarga moderada, assim denominadas no presente texto.

Observou-se associação significativa de sobrecarga com ocupação $\left(x^{2}=4,51 p=0,031\right)$, em que as cuidadoras aposentadas tinham maior sobrecarga que as que trabalhavam fora $(\mathrm{OR}=0,19)$. As pessoas que praticavam esporte relataram maior sobrecarga $\left(x^{2}=5,78 \mathrm{p}=\right.$ 0,008; OR= 5,92). Observou-se tendência de maior sobrecarga entre as pessoas que exerciam a função há mais de 60 meses $\left(x^{2}=3,31 \mathrm{p}=\right.$ 0,059; $\mathrm{OR}=4,02))$. Observou-se também maior sobrecarga nos que relataram ter religião $\left(x^{2}=\right.$ $4,75 \mathrm{p}=0,033 ; \mathrm{OR}=15,88)$, segundo as tabelas 1 e 2 .

Não foram encontradas associações significativas a outros hábitos de vida, nem a doenças autorreferidas. As variáveis relacionadas ao ato de cuidar não foram associadas à sobrecarga nessa população de estudo. Não foram observadas associações de sobrecarga ao grau de dependência do idoso, a transtornos mentais comuns, nem a queixas de memória (tabela 1).

Para a sobrecarga (Zarit), o modelo final conteve as variáveis: ocupação, esporte, religião e tempo na função de cuidador (tabela 2).

Tabela 1 - Resultado da análise bivariada entre os graus de sobrecarga da Escala Zarit e as variáveis independentes do estudo. Belo Horizonte, MG, 2010.

\begin{tabular}{|c|c|c|c|}
\hline \multirow[b]{2}{*}{ Variáveis } & \multicolumn{2}{|c|}{ Graus de Sobrecarga } & \multirow[b]{2}{*}{$\begin{array}{c}\text { Valor do Qui- } \\
\text { quadrado }\left(\mathrm{X}^{2}\right) \text { e de } p\end{array}$} \\
\hline & $\begin{array}{c}\text { Leve a Moderada } \\
30(51,72 \%)\end{array}$ & $\begin{array}{c}\text { Moderada a Severa } \\
28(48,28 \%)\end{array}$ & \\
\hline \multicolumn{4}{|l|}{ Idade } \\
\hline$<60$ anos & $19(63,3)$ & $18(64,3)$ & \\
\hline$\geq 60$ anos & $11(36,7)$ & $10(35,7)$ & $\chi^{2}=0,0057 \quad p=0,579$ \\
\hline \multicolumn{4}{|l|}{ Grau de Instrução } \\
\hline Até $2^{\circ}$ grau completo & $7(23,3)$ & $6(21,4)$ & \\
\hline $2^{\circ}$ grau completo & $9(30,0)$ & $9(32,1)$ & \\
\hline Ensino Superior & $14(46,7)$ & $13(46,4)$ & $\chi^{2}=0,045 \quad p=0,978$ \\
\hline \multicolumn{4}{|l|}{ Situação Conjugal } \\
\hline Com cônjuge & $17(56,7)$ & $12(42,9)$ & \\
\hline Sem cônjuge & $13(43,3)$ & $16(57,1)$ & $\chi^{2}=1,1048 \quad p=0,215$ \\
\hline \multicolumn{4}{|l|}{ Ocupaşão } \\
\hline Aposentado & $12(40,0)$ & $19(67,9)$ & \\
\hline Trabalhar fora & $18(60,0)$ & $9(32,1)$ & $\chi^{2}=4,511 \quad p=0,031^{*}$ \\
\hline \multicolumn{4}{|l|}{ Lazer } \\
\hline Não & $7(23,3)$ & $5(17,9)$ & \\
\hline $\operatorname{Sim}$ & $23(76,7)$ & $23(82,1)$ & $\chi^{2}=0,2647 \quad p=0,426$ \\
\hline
\end{tabular}




\begin{tabular}{|c|c|c|c|}
\hline \multirow[b]{2}{*}{ Variáveis } & \multicolumn{2}{|c|}{ Graus de Sobrecarga } & \multirow[b]{2}{*}{$\begin{array}{c}\text { Valor do Qui- } \\
\text { quadrado }\left(\mathrm{X}^{2}\right) \text { e de } p\end{array}$} \\
\hline & $\begin{array}{l}\text { Leve a Moderada } \\
\quad 30(51,72 \%)\end{array}$ & $\begin{array}{c}\text { Moderada a Severa } \\
28(48,28 \%)\end{array}$ & \\
\hline \multicolumn{4}{|l|}{ Prática de esporte } \\
\hline Não & $19(63,3)$ & $8(28,6)$ & \\
\hline $\operatorname{Sim}$ & $11(36,7)$ & $20(71,4)$ & $\chi^{2}=5,7841 p=0,008^{*}$ \\
\hline \multicolumn{4}{|l|}{ Média de horas de sono por noite } \\
\hline$\geq 8$ horas & $19(63,3)$ & $8(28,6)$ & \\
\hline$<8$ horas & $11(36,7)$ & $20(71,4)$ & $\chi^{2}=0,2222 \quad p=0,637$ \\
\hline \multicolumn{4}{|c|}{ Horas de sono/noite na última semana } \\
\hline$\geq 8$ horas & $9(30,0)$ & $9(32,1)$ & \\
\hline$<8$ horas & $21(70,0)$ & $19(67,9)$ & $\chi^{2}=0,0311 \quad p=0,542$ \\
\hline \multicolumn{4}{|l|}{ Fuma atualmente } \\
\hline Não fuma & $20(66,7)$ & $15(53,6)$ & \\
\hline Já fumou, mas não fuma & $9(30,0)$ & $11(39,3)$ & \\
\hline Fuma atualmente & $1(3,3)$ & $2(7,1)$ & $\chi^{2}=1,1801 \quad p=0,554$ \\
\hline \multicolumn{4}{|l|}{ Consumo atual de bebida alcoólica } \\
\hline Não bebe & $9(30,0)$ & $9(32,1)$ & \\
\hline Já bebeu, mas não bebe & $5(16,7)$ & $6(21,4)$ & \\
\hline Bebe atualmente & $16(53,3)$ & $13(46,4)$ & $\chi^{2}=0,8368 \quad p=0,847$ \\
\hline \multicolumn{4}{|l|}{ Ter religião } \\
\hline Não & $7(23,3)$ & $1(3,6)$ & \\
\hline $\operatorname{Sim}$ & $23(76,7)$ & $27(96,4)$ & $\chi^{2}=4,7567 \quad p=0,033^{*}$ \\
\hline \multicolumn{4}{|l|}{ Frequência a cultos } \\
\hline Não & $7(23,3)$ & $1(3,6)$ & \\
\hline Sim & $23(76,7)$ & $27(96,4)$ & $\chi^{2}=4,7567 \quad p=0,033^{*}$ \\
\hline
\end{tabular}

\section{DOENÇAS}

HAS

$\begin{array}{lccc}\text { Não } & 19(63,3) & 13(46,4) & \\ \text { Sim } & 11(36,7) & 15(53,6) & \chi^{2}=1,6734 \quad p=0,196 \\ \text { Diabetes } & & & \\ \text { Não } & 27(90,0) & 27(96,4) & \\ \text { Sim } & 3(10,0) & 1(3,6) & \chi^{2}=0,9321 \quad p=0,334 \\ \text { Insuficiência Renal } & & & \\ \text { Não } & 26(86,7) & 27(96,4) & \\ \text { Sim } & 4(13,3) & 1(3,6) & \chi^{2}=1,7520 \quad p=0,186\end{array}$




\begin{tabular}{|c|c|c|c|}
\hline \multirow[b]{2}{*}{ Variáveis } & \multicolumn{2}{|c|}{ Graus de Sobrecarga } & \multirow[b]{2}{*}{$\begin{array}{c}\text { Valor do Qui- } \\
\text { quadrado }\left(\mathrm{X}^{2}\right) \text { e de } p\end{array}$} \\
\hline & $\begin{array}{l}\text { Leve a Moderada } \\
\quad 30(51,72 \%)\end{array}$ & $\begin{array}{c}\text { Moderada a Severa } \\
28(48,28 \%)\end{array}$ & \\
\hline \multicolumn{4}{|l|}{ Anemia } \\
\hline Não & $20(66,7)$ & $20(71,4)$ & \\
\hline $\operatorname{Sim}$ & $10(33,3)$ & $8(28,6)$ & $\chi^{2}=0,1534 \quad p=0,695$ \\
\hline \multicolumn{4}{|c|}{ Insuficiência Cardíaca } \\
\hline Não & $28(93,3)$ & $25(89,3)$ & \\
\hline $\operatorname{Sim}$ & $2(6,7)$ & $3(10,7)$ & $\chi^{2}=0,3012 \quad p=0,583$ \\
\hline \multicolumn{4}{|c|}{ Derrame Cerebral (AVC) } \\
\hline Não & $30(100)$ & $26(92,9)$ & \\
\hline $\operatorname{Sim}$ & $0(0,0)$ & $2(7,1)$ & $\chi^{2}=2,2194 \quad p=0,136$ \\
\hline \multicolumn{4}{|c|}{ Colesterol Alto } \\
\hline Não & $16(53,3)$ & $12(42,9)$ & \\
\hline $\operatorname{Sim}$ & $14(46,7)$ & $16(57,1)$ & $\chi^{2}=0,6366 \quad p=0,425$ \\
\hline \multicolumn{4}{|c|}{ Dores nas costas ou problemas de coluna } \\
\hline Não & $10(33,3)$ & $11(39,3)$ & \\
\hline $\operatorname{Sim}$ & $20(66,7)$ & $17(60,7)$ & $\chi^{2}=0,2222 \quad p=0,637$ \\
\hline \multicolumn{4}{|c|}{ Dores articulares } \\
\hline Não & $11(36,7)$ & $12(42,9)$ & \\
\hline $\operatorname{Sim}$ & $19(63,3)$ & $16(57,1)$ & $\chi^{2}=0,2319 \quad p=0,630$ \\
\hline \multicolumn{4}{|c|}{ Asma ou bronquite } \\
\hline Não & $26(86,7)$ & $24(85,7)$ & \\
\hline $\operatorname{Sim}$ & $4(13,3)$ & $4(14,3)$ & $\chi^{2}=0,0110 \quad p=0,916$ \\
\hline \multicolumn{4}{|l|}{ Câncer } \\
\hline Não & $28(93,3)$ & $27(96,4)$ & \\
\hline Sim & $2(6,7)$ & $1(3,6)$ & $\chi^{2}=0,2829 \quad p=0,595$ \\
\hline \multicolumn{4}{|c|}{ MEDICAMENTOS } \\
\hline \multicolumn{4}{|c|}{ Medicação regular (últimos 7 dias) } \\
\hline Não & $3(10,0)$ & $2(7,1)$ & \\
\hline Sim & $27(90,0)$ & $26(92,9)$ & $\chi^{2}=0,1501 \quad p=0,698$ \\
\hline \multicolumn{4}{|c|}{ Antidepressivo } \\
\hline Não & $22(78,6)$ & $15(57,7)$ & \\
\hline $\operatorname{Sim}$ & $6(21,4)$ & $11(42,3)$ & $\chi^{2}=2,7246 \quad p=0,099$ \\
\hline \multicolumn{4}{|c|}{ Anticonvulsivante } \\
\hline Não & $28(100,0)$ & $23(88,5)$ & \\
\hline Sim & $0(0,0)$ & $3(11,5)$ & $\chi^{2}=2,7246 \quad p=0,099$ \\
\hline
\end{tabular}




\begin{tabular}{|c|c|c|c|}
\hline \multirow[b]{2}{*}{ Variáveis } & \multicolumn{2}{|c|}{ Graus de Sobrecarga } & \multirow[b]{2}{*}{$\begin{array}{c}\text { Valor do Qui- } \\
\text { quadrado }\left(\mathrm{X}^{2}\right) \text { e de } p\end{array}$} \\
\hline & $\begin{array}{c}\text { Leve a Moderada } \\
30(51,72 \%)\end{array}$ & $\begin{array}{c}\text { Moderada a Severa } \\
28(48,28 \%)\end{array}$ & \\
\hline \multicolumn{4}{|l|}{ Anti-bipertensivo } \\
\hline Não & $19(63,33)$ & $13(46,43)$ & \\
\hline Sim & $11(36,67)$ & $15(53,57)$ & $\chi^{2}=1,1136 \quad p=0,291$ \\
\hline \multicolumn{4}{|l|}{ Benzodiazepinico } \\
\hline Não & $26(92,9)$ & $24(92,3)$ & \\
\hline $\operatorname{Sim}$ & $2(7,1)$ & $2(7,7)$ & $\chi^{2}=0,0059 p=0,939$ \\
\hline \multicolumn{4}{|l|}{ Betabloqueador } \\
\hline Não & $28(100,0)$ & $23(88,5)$ & \\
\hline $\operatorname{Sim}$ & $0(0,0)$ & $3(11,5)$ & $\chi^{2}=3,4208 \quad p=0,064$ \\
\hline \multicolumn{4}{|l|}{ Ansiolitico } \\
\hline Não & $25(89,3)$ & $25(96,2)$ & \\
\hline $\operatorname{Sim}$ & $3(10,7)$ & $1(3,8)$ & $\chi^{2}=0,9272 \quad p=0,336$ \\
\hline \multicolumn{4}{|c|}{ Hormônio tireoidiano } \\
\hline Não & $22(78,6)$ & $21(80,8)$ & \\
\hline $\operatorname{Sim}$ & $6(21,4)$ & $5(19,2)$ & $\chi^{2}=0,0401 \quad p=0,841$ \\
\hline \multicolumn{4}{|l|}{ Corticoide } \\
\hline Não & $27(6,4)$ & $26(100)$ & \\
\hline $\operatorname{Sim}$ & $1(3,6)$ & $0(0,0)$ & $\chi^{2}=0,9461 \quad p=0,331$ \\
\hline \multicolumn{4}{|c|}{ Reposição hormonal } \\
\hline Não & $25(89,3)$ & $24(92,3)$ & \\
\hline Sim & $3(10,7)$ & $2(7,7)$ & $\chi^{2}=0,1465 \quad p=0,702$ \\
\hline \multicolumn{4}{|c|}{ Consulta médica (últimos 12 meses) } \\
\hline Não & $2(6,7)$ & $1(3,6)$ & \\
\hline Sim & $28(93,3$ & $27(96,4)$ & $\chi^{2}=0,2829 p=0,595$ \\
\hline \multicolumn{4}{|c|}{ ATO DE CUIDAR } \\
\hline \multicolumn{4}{|c|}{ Grau de parentesco } \\
\hline Esposa & $5(16,7)$ & $6(21,4)$ & \\
\hline Filha(o) & $22(73,3)$ & $20(71,4)$ & \\
\hline Outros & $3(10,0)$ & $2(7,1)$ & $\chi^{2}=0,318 \quad p=0,853$ \\
\hline \multicolumn{4}{|c|}{ Tempo diário na função de cuidador } \\
\hline$<24$ horas & $13(43,3)$ & $16(57,1)$ & \\
\hline$=24$ horas & $17(56,7)$ & $12(42,9)$ & $\chi^{2}=1,1048 \quad p=0,215$ \\
\hline
\end{tabular}




\begin{tabular}{|c|c|c|c|}
\hline \multirow[b]{2}{*}{ Variáveis } & \multicolumn{2}{|c|}{ Graus de Sobrecarga } & \multirow[b]{2}{*}{$\begin{array}{c}\text { Valor do Qui- } \\
\text { quadrado }\left(\mathrm{X}^{2}\right) \text { e de } p\end{array}$} \\
\hline & $\begin{array}{l}\text { Leve a Moderada } \\
\quad 30(51,72 \%)\end{array}$ & $\begin{array}{c}\text { Moderada a Severa } \\
28(48,28 \%)\end{array}$ & \\
\hline \multicolumn{4}{|l|}{ Tempo na função de cuidador } \\
\hline$<60$ meses & $20(66,7)$ & $12(42,9)$ & \\
\hline$\geq 60$ meses & $10(33,3)$ & $16(57,1)$ & $\chi^{2}=3,3196$ \\
\hline \multicolumn{4}{|l|}{ Tempo de diagnóstico da demência } \\
\hline$<60$ meses & $18(60,0)$ & $11(39,3)$ & \\
\hline$\geq 60$ meses & $12(40,0)$ & $17(60,7)$ & $\chi^{2}=2,4857 \quad p=0,094$ \\
\hline \multicolumn{4}{|l|}{ Escala de Pfeffer } \\
\hline$<30$ (parcialmente dependente) & $19(63,3)$ & $12(42,9)$ & \\
\hline$=30$ (totalmente dependente) & $11(36,7)$ & $16(57,1)$ & $\chi^{2}=2,4405 \quad p=0,097$ \\
\hline \multicolumn{4}{|l|}{ Transtornos mentais comuns } \\
\hline$\leq 7$ & $13(43,3)$ & $18(64,3)$ & \\
\hline$>7$ & $17(56,7)$ & $10(35,7)$ & $\chi^{2}=2,5553 \quad p=0,091$ \\
\hline \multicolumn{4}{|l|}{ Queixa de memória (PRMQ) } \\
\hline$<20$ & $9(30,0)$ & $9(32,1)$ & \\
\hline$>21$ & $21(70,0)$ & $19(67,9)$ & $\chi^{2}=0,0311 \quad p=0,542$ \\
\hline
\end{tabular}

Tabela 2 - Resultado da análise de regressão logística múltipla das características associadas à sobrecarga das cuidadoras. Belo Horizonte, MG, 2010.

\begin{tabular}{lccc}
\hline \multicolumn{1}{c}{ Escala de Sobrecarga Zarit } & $\begin{array}{c}\text { Estimativa do } \\
\text { Risco (OR)* }\end{array}$ & Valor de p & $\begin{array}{c}\text { Intervalo de } \\
\text { Confiança (95\%) }\end{array}$ \\
\hline Trabalhar fora & 0,19 & 0,014 & $0,05-0,71$ \\
Prática de esporte(sim) & 5,92 & 0,010 & $1,53-22,91$ \\
Ter religião (sim) & 15,88 & 0,032 & $1,28-197,59$ \\
Tempo na função de cuidador ( $\geq 60$ meses) & 4,02 & 0,046 & $1,03-15,70$ \\
\hline
\end{tabular}

*Ajustado pelas variáveis $(\mathrm{p}<0,2)$ : ocupação, prática de esporte, ter religião, frequência a cultos, HAS, insuficiência renal, derrame cerebral, antidepressivo, anticonvulsivante, betabloqueador, tempo na função de cuidador, tempo de diagnóstico da demência, escala de Pfeffer e transtornos mentais comuns.

Vinte e sete $(46,55 \%)$ cuidadoras apresentaram ansiedade/depressão. Houve associação significativa entre esses transtornos mentais comuns e queixas de dores nas costas $\left(x^{2}=4,27 \mathrm{p}=0,035\right)$, uso de benzodiazepínicos $\left(x^{2}=4,65 \mathrm{p}=0,047\right)$ e tendência à significância para ser cuidadora filha $\left(x^{2}=5,66 \mathrm{p}=0,059\right)$, segundo a tabela 3 . 
Tabela 3 - Resultado da análise bivariada entre os transtornos mentais comuns e as variáveis independentes do estudo. Belo Horizonte, MG, 2010.

\begin{tabular}{|c|c|c|c|}
\hline \multirow{3}{*}{ Variáveis } & \multicolumn{3}{|c|}{ Transtornos mentais comuns } \\
\hline & AUSENTES & PRESENTES & Valor do Qui-quadrado \\
\hline & $31(53,45 \%)$ & $27(46,55 \%)$ & $\left(\mathrm{X}^{2}\right)$ e de $p$ \\
\hline \multicolumn{4}{|l|}{ Idade } \\
\hline$<60$ anos & $22(71,0)$ & $15(55,6)$ & \\
\hline$\geq 60$ anos & $9(29,0)$ & $12(44,4)$ & $\chi^{2}=1,4841 \quad p=0,173$ \\
\hline \multicolumn{4}{|l|}{ Grau de Instrução } \\
\hline Até $2^{\circ}$ grau completo & $9(29,0)$ & $4(14,8)$ & \\
\hline $2^{\circ}$ grau completo & $9(29,1)$ & $9(33,3)$ & \\
\hline Ensino Superior & $13(41,9)$ & $14(51,9)$ & $\chi^{2}=1,6923 \quad p=0,429$ \\
\hline \multicolumn{4}{|l|}{ Situação Conjugal } \\
\hline Com cônjuge & $14(45,2)$ & $15(55,6)$ & \\
\hline Sem cônjuge & $17(54,8)$ & $12(44,4)$ & $\chi^{2}=0,6237 \quad p=0,430$ \\
\hline \multicolumn{4}{|l|}{ Ocupação } \\
\hline Aposentado & $17(54,8)$ & $14(51,9)$ & \\
\hline Trabalhar fora & $14(45,2)$ & $13(48,1)$ & $\chi^{2}=0,0517 \quad p=0,820$ \\
\hline \multicolumn{4}{|l|}{ Lazer } \\
\hline Não & $5(16,1)$ & $7(25,9)$ & \\
\hline $\operatorname{Sim}$ & $26(83,9)$ & $20(74,1)$ & $\chi^{2}=0,8441 \quad p=0,276$ \\
\hline \multicolumn{4}{|l|}{ Prática de esporte } \\
\hline Não & $11(35,5)$ & $16(59,3)$ & \\
\hline $\operatorname{Sim}$ & $20(64,5)$ & $11(40,7)$ & $\chi^{2}=2,3507 \quad p=0,061$ \\
\hline \multicolumn{4}{|l|}{ Média de horas de sono por noite } \\
\hline$\geq 8$ horas & $12(38,7)$ & $9(33,3)$ & \\
\hline$<8$ horas & $19(61,3)$ & $18(66,7)$ & $\chi^{2}=0,1806 \quad p=0,671$ \\
\hline \multicolumn{4}{|c|}{ Horas de sono/noite na última semana } \\
\hline$\geq 8$ horas & & $9(33,3)$ & \\
\hline$<8$ horas & $22(71,0)$ & $18(66,7)$ & $\chi^{2}=0,0311 \quad p=0,860$ \\
\hline \multicolumn{4}{|l|}{ Fuma atualmente } \\
\hline Não fuma & $20(64,5)$ & $15(55,6)$ & \\
\hline Já fumou, mas não fuma & $9(29,0)$ & $11(40,7)$ & \\
\hline Fuma atualmente & $2(6,5)$ & $1(3,7)$ & $\chi^{2}=0,9764 \quad p=0,614$ \\
\hline
\end{tabular}




\begin{tabular}{lccc}
\hline \multirow{2}{*}{ Variáveis } & \multicolumn{3}{c}{ Transtornos mentais comuns } \\
\cline { 2 - 4 } & AUSENTES & PRESENTES & Valor do Qui-quadrado \\
\cline { 2 - 5 } & $31(53,45 \%)$ & $27(46,55 \%)$ & $\left(\mathrm{X}^{2}\right)$ e de $p$ \\
\hline Consumo atual de bebida alcoólica & $12(38,7)$ & $6(22,2)$ & \\
Não bebe & $7(22,6)$ & $4(14,8)$ & \\
Já bebeu, mas não bebe & $12(38,7)$ & $17(63,0)$ & $\chi^{2}=2,6415 \quad p=0,181$ \\
Bebe atualmente & & & \\
Ter religião & $3(9,7)$ & $5(18,5)$ & \\
Não & $28(90,3)$ & $22(81,5)$ & $\chi^{2}=0,9486 \quad p=0,277$ \\
Sim & & & \\
Frequência a cultos & $5(16,1)$ & $6(22,2)$ & \\
Não & $26(83,9)$ & $21(77,8)$ & $\chi^{2}=0,5145 \quad p=0,398$ \\
Sim & &
\end{tabular}

\section{DOENÇAS}

$H A S$

Não

Sim

Diabetes

Não

Sim

Insuficiência Renal

Não

Sim

Anemia

Não

Sim

Insuficiência Cardíaca

Não

Sim

Derrame Cerebral (AVC)

Não

Sim

Colesterol Alto

Não

Sim
$19(61,3)$

$12(38,7)$

$30(96,8)$

$1(3,2)$

$29(93,5)$

$$
2(6,5)
$$

$23(74,2)$

$8(25,8)$

$28(90,3)$

$3(9,7)$

$30(96,8)$

$1(3,2)$

$15(48,4)$

$16(51,6)$
$13(48,1)$

$14(51,9)$

$\chi^{2}=1,0078 \quad p=0,315$

$24(88,9)$

$3(11,1)$

$\chi^{2}=1,3975 \quad p=0,255$

$24(88,9)$

$3(11,1)$

$\chi^{2}=0,3977 \quad p=0,433$

$17(63,0)$

$10(37,0)$

$\chi^{2}=0,8504 \quad p=0,262$

$25(92,6)$

$2(7,4)$

$\chi^{2}=0,0944 \quad p=0,759$

$26(96,3)$

$1(3,7)$

$\chi^{2}=0,0099 \quad p=0,921$

$13(48,1)$

$14(51,9)$ 


\begin{tabular}{|c|c|c|c|}
\hline \multirow{3}{*}{ Variáveis } & \multicolumn{3}{|c|}{ Transtornos mentais comuns } \\
\hline & AUSENTES & PRESENTES & Valor do Qui-quadrado \\
\hline & $31(53,45 \%)$ & $27(46,55 \%)$ & $\left(\mathrm{X}^{2}\right)$ e de $p$ \\
\hline \multicolumn{4}{|c|}{ Dores nas costas ou problemas de coluna } \\
\hline Não & $15(48,4)$ & $6(22,2)$ & \\
\hline $\operatorname{Sim}$ & $16(51,6)$ & $21(77,8)$ & $\chi^{2}=4,2773 p=0,035^{*}$ \\
\hline \multicolumn{4}{|l|}{ Dores articulares } \\
\hline Não & $13(41,9)$ & $10(37,0)$ & \\
\hline $\operatorname{Sim}$ & $18(58,1)$ & $17(63,0)$ & $\chi^{2}=0,1447 \quad p=0,456$ \\
\hline \multicolumn{4}{|l|}{ Asma ou bronquite } \\
\hline Não & $27(87,1)$ & $23(85,2)$ & \\
\hline Sim & $4(12,9)$ & $4(14,8)$ & $\chi^{2}=0,0443 \quad p=0,565$ \\
\hline \multicolumn{4}{|l|}{ Câncer } \\
\hline Não & $30(96,8)$ & $25(92,6)$ & \\
\hline $\operatorname{Sim}$ & $1(3,2)$ & $2(7,4)$ & $\chi^{2}=0,5145 \quad p=0,447$ \\
\hline \multicolumn{4}{|l|}{ MEDICAMENTOS } \\
\hline \multicolumn{4}{|c|}{ Medicação regular (ültimos 7 dias) } \\
\hline Não & $3(9,7)$ & $2(7,4)$ & \\
\hline Sim & $28(90,3)$ & $25(92,6)$ & $\chi^{2}=0,0944 \quad p=0,567$ \\
\hline \multicolumn{4}{|l|}{ Antidepressivo } \\
\hline Não & $21(75,0)$ & $16(61,5)$ & \\
\hline $\operatorname{Sim}$ & $7(25,0)$ & $10(38,5)$ & $\chi^{2}=1,1326 \quad p=0,287$ \\
\hline \multicolumn{4}{|l|}{ Anti-hipertensivo } \\
\hline Não & $17(60,7)$ & $16(61,5)$ & \\
\hline $\operatorname{Sim}$ & $11(39,3)$ & $10-38,5$ & $\chi^{2}=0,0039 \quad p=0,951$ \\
\hline \multicolumn{4}{|l|}{ Anticonvulsivante } \\
\hline Não & $27(96,4)$ & $24(92,3)$ & \\
\hline $\operatorname{Sim}$ & $1(3,6)$ & $2(7,7)$ & $\chi^{2}=0,4363 \quad p=0,472$ \\
\hline \multicolumn{4}{|l|}{ Benzodiazepínico } \\
\hline Não & $28(100,0)$ & $22(84,6)$ & \\
\hline $\operatorname{Sim}$ & $0(0,0)$ & $4(15,4)$ & $\chi^{2}=4,6523 p=0,047^{*}$ \\
\hline \multicolumn{4}{|l|}{ Betabloqueador } \\
\hline Não & $26(92,9)$ & $25(96,2)$ & \\
\hline $\operatorname{Sim}$ & $2(7,1)$ & $1(3,8)$ & $\chi^{2}=0,2793 \quad p=0,528$ \\
\hline
\end{tabular}




\begin{tabular}{|c|c|c|c|}
\hline \multirow{3}{*}{ Variáveis } & \multicolumn{3}{|c|}{ Transtornos mentais comuns } \\
\hline & AUSENTES & PRESENTES & Valor do Qui-quadrado \\
\hline & $31(53,45 \%)$ & $27(46,55 \%)$ & $\left(\mathrm{X}^{2}\right)$ e de $p$ \\
\hline \multicolumn{4}{|l|}{ Ansiolítico } \\
\hline Não & $26(92,9)$ & $24(92,3)$ & \\
\hline Sim & $2(7,1)$ & $2(7,7)$ & $\chi^{2}=0,0059 \quad p=0,666$ \\
\hline \multicolumn{4}{|l|}{ Hormônio tireoidiano } \\
\hline Não & $21(75,0)$ & $22(84,6)$ & \\
\hline $\operatorname{Sim}$ & $7(25,0)$ & $4(15,4)$ & $\chi^{2}=0,7684 \quad p=0,381$ \\
\hline \multicolumn{4}{|l|}{ Corticoide } \\
\hline Não & $28(100,0)$ & $25(96,2)$ & \\
\hline Sim & $0(0,0)$ & $1(3,8)$ & $\chi^{2}=1,0972 \quad p=0,295$ \\
\hline \multicolumn{4}{|l|}{ Reposição hormonal } \\
\hline Não & $25(89,3)$ & $24(92,3)$ & \\
\hline $\operatorname{Sim}$ & $3(10,7)$ & $2(7,7)$ & $\chi^{2}=0,5145 \quad p=0,536$ \\
\hline \multicolumn{4}{|c|}{ Consulta médica (últimos 12 meses) } \\
\hline Não & $2(6,5)$ & $1(3,7)$ & \\
\hline $\operatorname{Sim}$ & $29(93,5)$ & $26(96,3)$ & $\chi^{2}=0,2829 \quad p=0,595$ \\
\hline \multicolumn{4}{|l|}{ ATO DE CUIDAR } \\
\hline \multicolumn{4}{|l|}{ Grau de parentesco } \\
\hline Esposa & $4(12,9)$ & $7(25,9)$ & \\
\hline Filha(o) & $22(71,0)$ & $20(74,1)$ & \\
\hline Outros & $5(16,1)$ & $0(0,0)$ & $\chi^{2}=5,6645 p=0,059^{*}$ \\
\hline \multicolumn{4}{|c|}{ Tempo diário na função de cuidador } \\
\hline$<24$ horas & $13(41,9)$ & $16(59,3)$ & \\
\hline$=24$ horas & $18(58,1)$ & $11(40,7)$ & $\chi^{2}=1,7324 \quad p=0,146$ \\
\hline \multicolumn{4}{|l|}{ Tempo na função de cuidador } \\
\hline$<60$ meses & $15(48,4)$ & $17(63,0)$ & $\chi^{2}=1,2396 \quad p=0,198$ \\
\hline$\geq 60$ meses & $16(51,6)$ & $10(37,0)$ & \\
\hline \multicolumn{4}{|c|}{ Tempo de diagnóstico da demência } \\
\hline$<60$ meses & $15(48,4)$ & $14(51,9)$ & \\
\hline$\geq 60$ meses & $16(51,6)$ & $13(48,1)$ & $\chi^{2}=0,871 p=0,500$ \\
\hline \multicolumn{4}{|c|}{ Grau de dependência na Escala Zarit } \\
\hline Leve a moderada & $18(58,1)$ & $10(37,0)$ & \\
\hline Moderada a Severa & $13(41,9)$ & $17(63,0)$ & $\chi^{2}=2,5553 p=0,110$ \\
\hline
\end{tabular}




\begin{tabular}{lccc}
\hline \multirow{2}{*}{ Variáveis } & \multicolumn{3}{c}{ Transtornos mentais comuns } \\
\cline { 2 - 4 } & AUSENTES & PRESENTES & Valor do Qui-quadrado \\
\cline { 2 - 4 } & $31(53,45 \%)$ & $27(46,55 \%)$ & $\left(\mathrm{X}^{2}\right)$ e de $p$ \\
\hline Escala de Pfeffer & & & \\
$<30$ (parcialmente dependente) & $15(48,4)$ & $16(59,3)$ & \\
$=30$ (totalmente dependente) & $16(51,6)$ & $11(40,7)$ & $\chi^{2}=0,6856 p=0,287$ \\
Queixa de memória (PRMQ) & & & \\
$<20$ & $12(38,7)$ & $6(22,2)$ & \\
$>21$ & $19(61,3)$ & $21(77,8)$ & $\chi^{2}=1,832 p=0,142$ \\
\hline$*=p<0,05$ & & &
\end{tabular}

O modelo final apresentou associação com esporte, dores nas costas e tempo na função de cuidador (tabela 4).

Tabela 4 - Análise de regressão logística múltipla das características associadas aos transtornos mentais comuns. Belo Horizonte, MG, 2010.

\begin{tabular}{lccc}
\hline \multicolumn{1}{c}{ Transtornos mentais comuns } & $\begin{array}{c}\text { Estimativa de } \\
\text { risco (OR)* }\end{array}$ & Valor de p & $\begin{array}{c}\text { Intervalo de } \\
\text { Confiança (95\%) }\end{array}$ \\
\hline Esporte (sim) & 0,2 & 0,028 & $0,07-0,86$ \\
Dores nas costas (sim) & 4,24 & 0,026 & $1,18-15,15$ \\
Tempo diário na função de cuidador (=24 h) & 0,25 & 0,037 & $0,70-0,92$ \\
\hline
\end{tabular}

*Ajustado pelas variáveis $(\mathrm{p}<0,2)$ : idade, prática de esporte,consumo de bebida alcoólica, dores nas costas, benzodiazepínicos, grau de parentesco, tempo diário na função de cuidador, tempo na função de cuidador, escala Zarit e queixa de memória.

Tabela 5 - Resultado da análise bivariada entre as queixas de memória e as variáveis independentes do estudo. Belo Horizonte, MG, 2010.

\begin{tabular}{|c|c|c|c|}
\hline \multirow[b]{2}{*}{ Variáveis } & \multicolumn{2}{|c|}{ Queixa de memória } & \multirow[b]{2}{*}{$\begin{array}{c}\text { Valor do Qui- } \\
\text { quadrado }\left(\mathrm{X}^{2}\right) \text { e de } \mathrm{p}\end{array}$} \\
\hline & $\begin{array}{c}\text { MENOS } \\
\text { FREQUENTE } \\
<2018(31,03 \%)\end{array}$ & $\begin{array}{c}\text { MUITO } \\
\text { FREQUENTE } \\
\geq 20 \\
40(68,97 \%)\end{array}$ & \\
\hline \multicolumn{4}{|l|}{ Idade } \\
\hline$<60$ anos & $12(66,7)$ & $25(62,5)$ & \\
\hline$\geq 60$ anos & $6(33,3)$ & $15(37,5)$ & $\chi^{2}=0,0933 \quad p=0,760$ \\
\hline \multicolumn{4}{|l|}{ Grau de Instrução } \\
\hline Até $2^{\circ}$ grau completo & $5(27,8)$ & $8(20,0)$ & \\
\hline $2^{\circ}$ grau completo & $6(33,3)$ & $12(30,0)$ & \\
\hline Ensino Superior & $7(38,9)$ & $20(50,0)$ & $\chi^{2}=0,7087 \quad p=0,702$ \\
\hline
\end{tabular}




\begin{tabular}{|c|c|c|c|}
\hline \multirow[b]{2}{*}{ Variáveis } & \multicolumn{2}{|c|}{ Queixa de memória } & \\
\hline & $\begin{array}{c}\text { MENOS } \\
\text { FREQUENTE } \\
<2018(31,03 \%)\end{array}$ & $\begin{array}{c}\text { MUITO } \\
\text { FREQUENTE } \\
\geq 20 \\
40(68,97 \%)\end{array}$ & $\begin{array}{c}\text { Valor do Qui- } \\
\text { quadrado }\left(\mathrm{X}^{2}\right) \text { e de } \mathrm{p}\end{array}$ \\
\hline \multicolumn{4}{|l|}{ Situação Conjugal } \\
\hline Com cônjuge & $8(44,4)$ & $21(52,5)$ & \\
\hline Sem cônjuge & $10(55,6)$ & $19(47,5)$ & $\chi^{2}=0,3222 \quad p=0,570$ \\
\hline \multicolumn{4}{|l|}{ Ocupação } \\
\hline Aposentado & $11(61,1)$ & $20(50,0)$ & \\
\hline Trabalhar fora & $7(38,9)$ & $20(50,0)$ & $\chi^{2}=0,6160 \quad p=0,433$ \\
\hline \multicolumn{4}{|l|}{ Lazer } \\
\hline Não & $4(22,2)$ & $8(20,0)$ & \\
\hline $\operatorname{Sim}$ & $14(77,8)$ & $32(80,0)$ & $\chi^{2}=0,0374 \quad p=0,8$ \\
\hline \multicolumn{4}{|l|}{ Prática de esporte } \\
\hline Não & $8(44,4)$ & $19(47,5)$ & \\
\hline $\operatorname{Sim}$ & $10(55,6)$ & $21(52,5)$ & $\chi^{2}=0,0015 \quad p=0,969$ \\
\hline \multicolumn{4}{|l|}{ Média de horas de sono por noite } \\
\hline$\geq 8$ horas & $8(44,4)$ & $13(32,5)$ & \\
\hline$<8$ horas & $10(55,6)$ & $27(67,5)$ & $\chi^{2}=0,7668 \quad p=0,381$ \\
\hline \multicolumn{4}{|c|}{ Horas de sono/noite na última semana } \\
\hline $\begin{array}{l}\geq 8 \text { horas } \\
<8 \text { horas }\end{array}$ & $\begin{array}{c}6(33,3) \\
12(66,7)\end{array}$ & $\begin{array}{l}12(30,0) \\
28(70,0)\end{array}$ & $\chi^{2}=0,0644 \quad p=0,800$ \\
\hline \multicolumn{4}{|l|}{ Fuma atualmente } \\
\hline Não fuma & $9(50,0)$ & $26(65,0)$ & \\
\hline Já fumou, mas não fuma & $8(44,4)$ & $12(30,0)$ & \\
\hline Fuma atualmente & $1(5,6)$ & $2(5,0)$ & $\chi^{2}=1,2214 p=0,543$ \\
\hline \multicolumn{4}{|l|}{ Consumo atual de bebida alcoólica } \\
\hline Não bebe & $5(27,8)$ & $13(32,5)$ & \\
\hline Já bebeu, mas não bebe & $4(22,2)$ & $7(17,5)$ & \\
\hline Bebe atualmente & $9(50,0)$ & $20(50,0)$ & $\chi^{2}=0,1349 \quad p=0,935$ \\
\hline \multicolumn{4}{|l|}{ Ter religião } \\
\hline Não & $0(0,0)$ & $8(20,0)$ & \\
\hline $\operatorname{Sim}$ & $18(100,0)$ & $32(80,0)$ & $\chi^{2}=4,1760 \quad p=0,041^{*}$ \\
\hline \multicolumn{4}{|l|}{ Frequência a cultos } \\
\hline Não & $0(0,0)$ & $11(27,5)$ & \\
\hline $\operatorname{Sim}$ & $18(100,0)$ & $29(72,5)$ & $\chi^{2}=6,1085 p=0,013^{*}$ \\
\hline
\end{tabular}




\begin{tabular}{lccc}
\hline \multirow{2}{*}{ Variáveis } & Queixa de memória & \\
\cline { 2 - 3 } & FREQUENTE & MUITO & Valor do Qui- \\
& $<2018(31,03 \%)$ & $\geq 20$ & quadrado $\left(\mathrm{X}^{2}\right)$ e de $\mathrm{p}$ \\
& & $40(68,97 \%)$ & \\
\hline
\end{tabular}

\section{DOENÇAS}

$H A S$

$$
\begin{aligned}
& \text { Não } \\
& \text { Sim }
\end{aligned}
$$

Diabetes

Não

Sim

Insuficiência Renal

Não

Sim

Anemia

Não

Sim

Insuficiência Cardíaca

Não

Sim

Derrame Cerebral (AVC)

Não

Sim

Colesterol Alto

Não

Sim

Dores nas costas ou problemas de coluna

Não

Sim

Dores articulares

Não

Sim

Asma ou bronquite

Não

Sim

Câncer

Não

Sim

$$
9(50,0)
$$

$9(50,0)$

$16(88,9)$

$2(11,1)$

$18(100,0)$

$0(0,0)$

$15(83,3)$

$3(16,7)$

$15(83,3)$

$3(16,7)$

$18(100,0)$

$0(0,0)$

$9(50,0)$

$9(50,0)$

$7(38,9)$

$11(61,1)$

$8(44,4)$

$10(55,6)$

$15(83,3)$

$3(16,7)$

$18(100,0)$

$0(0,0)$
$23(57,5)$

$17(42,5)$

$\chi^{2}=0,2823 \quad p=0,595$

$38(95,0)$

$2(5,0)$

$\chi^{2}=0,7220 \quad p=0,395$

$35(87,5)$

$5(12,5)$

$\chi^{2}=2,4623 \quad p=0,117$

$25(62,5)$

$15(37,5)$

$\chi^{2}=2,5174 \quad p=0,110$

$38(95,0)$

$2(5,0)$

$\chi^{2}=2,1449 \quad p=0,143$

$38(95,0)$

$2(5,0)$

$\chi^{2}=0,9321 \quad p=0,334$

$19(47,5)$

$21(52,5)$

$\chi^{2}=0,0311 \quad p=0,860$

$14(35,0)$

$26(65,0)$

$\chi^{2}=0,0813 p=0,776$

$15(37,5)$

$25(62,5)$

$\chi^{2}=0,2502 \quad p=0,617$

$35(87,5)$

$5(12,5)$

$\chi^{2}=0,1812 p=0,670$

$37(92,5)$

$3(7,5)$ 


\begin{tabular}{lccc}
\hline \multirow{4}{*}{ Variáveis } & \multicolumn{3}{c}{ Queixa de memória } \\
\cline { 2 - 4 } & MENOS & MUITO & Valor do Qui- \\
& FREQUENTE & FREQUENTE & quadrado(X ${ }^{2}$ e de p \\
& $<2018(31,03 \%)$ & $\geq 20$ & \\
& & $40(68,97 \%)$ & \\
\hline
\end{tabular}

\section{MEDICAMENTOS}

Medicação regular (últimos 7 dias)

\begin{tabular}{|c|c|c|c|}
\hline Não & $1(5,6)$ & $4(10,0)$ & \\
\hline $\operatorname{Sim}$ & $17(94,4)$ & $36(90,0)$ & $\chi^{2}=0,3113 \quad p=0,577$ \\
\hline \multicolumn{4}{|c|}{ Antidepressivo } \\
\hline Não & $15(88,2)$ & $22(59,5)$ & \\
\hline Sim & $2(11,8)$ & $15(40,5)$ & $\chi^{2}=4,4715 \quad p=0,034^{*}$ \\
\hline \multicolumn{4}{|c|}{ Anti-bipertensivo } \\
\hline Não & $8(47,1)$ & $25(67,6)$ & \\
\hline $\operatorname{Sim}$ & $9(52,9)$ & $12(32,4)$ & $\chi^{2}=0,6160 \quad p=0,433$ \\
\hline \multicolumn{4}{|c|}{ Anticonvulsivante } \\
\hline Não & $17(100,0)$ & $34(91,9)$ & \\
\hline Sim & $0(0,0)$ & $3(8,1)$ & $\chi^{2}=1,4595 \quad p=0,227$ \\
\hline \multicolumn{4}{|c|}{ Benzodiazepinico } \\
\hline Não & $16(94,1)$ & $34(91,9)$ & \\
\hline Sim & $1(5,9)$ & $3(8,1)$ & $\chi^{2}=0,0841 \quad p=0,772$ \\
\hline \multicolumn{4}{|c|}{ Betabloqueador } \\
\hline Não & $17(100,0)$ & $34(91,9)$ & \\
\hline Sim & $0(0,0)$ & $3(8,1)$ & $\chi^{2}=1,4595 \quad p=0,227$ \\
\hline \multicolumn{4}{|c|}{ Ansiolítico } \\
\hline Não & $16(94,1)$ & $34(91,9)$ & \\
\hline $\operatorname{Sim}$ & $1(5,9)$ & $3(8,1)$ & $\chi^{2}=0,841 \quad p=0,772$ \\
\hline \multicolumn{4}{|c|}{ Hormônio tireoidiano } \\
\hline Não & $12(70,6)$ & $31(83,8)$ & \\
\hline $\operatorname{Sim}$ & $5(29,4)$ & $6(16,2)$ & $\chi^{2}=1,2504 \quad p=0,263$ \\
\hline \multicolumn{4}{|c|}{ Corticoide } \\
\hline Não & $16(94,1)$ & $37(100,0)$ & \\
\hline Sim & $1(5,9)$ & $0(0,0)$ & $\chi^{2}=2,2175 \quad p=0,136$ \\
\hline \multicolumn{4}{|c|}{ Reposição Hormonal } \\
\hline Não & $15(88,2)$ & $34(91,9)$ & \\
\hline $\operatorname{Sim}$ & $2(11,8)$ & $3(8,1)$ & $\chi^{2}=0,1854 \quad p=0,667$ \\
\hline
\end{tabular}




\begin{tabular}{|c|c|c|c|}
\hline \multirow[b]{2}{*}{ Variáveis } & \multicolumn{2}{|c|}{ Queixa de memória } & \multirow[b]{2}{*}{$\begin{array}{c}\text { Valor do Qui- } \\
\text { quadrado }\left(\mathrm{X}^{2}\right) \text { e de } \mathrm{p}\end{array}$} \\
\hline & $\begin{array}{c}\text { MENOS } \\
\text { FREQUENTE } \\
<2018(31,03 \%)\end{array}$ & $\begin{array}{c}\text { MUITO } \\
\text { FREQUENTE } \\
\geq 20 \\
40(68,97 \%)\end{array}$ & \\
\hline \multicolumn{4}{|l|}{ Consulta médica (últimos 12 meses) } \\
\hline Não & $0(0,0)$ & $3(7,5)$ & \\
\hline $\operatorname{Sim}$ & $18(100,0)$ & $37(92,5)$ & $\chi^{2}=0,2820 p=0,595$ \\
\hline \multicolumn{4}{|l|}{ ATO DE CUIDAR } \\
\hline \multicolumn{4}{|l|}{ Grau de parentesco } \\
\hline Esposa & $5(27,8)$ & $6(15,0)$ & \\
\hline Filha(o) & $11(61,1)$ & $31(77,5)$ & \\
\hline Outros & $2(11,1)$ & $3(7,5)$ & $\chi^{2}=1,7169 \quad p=0,424$ \\
\hline \multicolumn{4}{|l|}{ Tempo diário na função de cuidador } \\
\hline$<24$ horas & $6(33,3)$ & $23(57,5)$ & \\
\hline$=24$ horas & $12(66,7)$ & $17(42,5)$ & $\chi^{2}=2,9000 \quad p=0,089$ \\
\hline \multicolumn{4}{|l|}{ Tempo na função de cuidador } \\
\hline$<60$ meses & $9(50,0)$ & $23(57,5)$ & \\
\hline$\geq 60$ meses & $9(50,0)$ & $17(42,5)$ & $\chi^{2}=0,2823 \quad p=0,595$ \\
\hline \multicolumn{4}{|l|}{ Tempo de diagnóstico da demência } \\
\hline$<60$ meses & $8(44,4)$ & $21(52,5)$ & \\
\hline$\geq 60$ meses & $10(55,6)$ & $19(47,5)$ & $\chi^{2}=0,3222 \quad p=0,570$ \\
\hline \multicolumn{4}{|l|}{ Transtornos mentais comuns } \\
\hline$\leq 7$ & $12(66,7)$ & $19(47,5)$ & \\
\hline$>7$ & $6(33,3)$ & $21(52,5)$ & $\chi^{2}=1,8329 \quad p=0,176$ \\
\hline \multicolumn{4}{|l|}{ Grau de sobrecarga na escala Zarit } \\
\hline Leve a moderada & $9(50,0)$ & $21(52,5)$ & \\
\hline Moderada a severa & $9(50,0)$ & $19(47,5)$ & $\chi^{2}=0,0311 \quad p=0,860$ \\
\hline \multicolumn{4}{|l|}{ Escala de Pfeffer } \\
\hline$<30$ (parcialmente dependente) & $12(66,7)$ & $19(47,5)$ & \\
\hline$=30$ (totalmente dependente) & $6(33,3)$ & $21(52,5)$ & $\chi^{2}=1,8329 \quad p=0,176$ \\
\hline
\end{tabular}

$*=P<0,05$ 
A maioria das cuidadoras, 40 (68,97\%), apresentava queixas de memória. Foi observada associação da presença da queixa ao uso de antidepressivo $(p=0,034)$, em que $40,5 \%$ das usuárias apresentaram mais queixas subjetivas de memória. As participantes que alegaram ter religião apresentaram maior frequência de queixas de memória $\left(\chi^{2}=4,1760 p=\right.$ 0,041), assim como ir à missa $\left(\chi^{2}=6,1085 p\right.$ $=0,013)$, segundo a tabela 5. Após ajuste pelas variáveis $(p<0,2)$, como ter religião, frequência a cultos, insuficiência renal, anemia, insuficiência cardíaca, uso de antidepressivo, corticoide, tempo diário na função de cuidador, transtornos mentais comuns e escala de Pfeffer, essas associações não mantiveram significância estatística no modelo final.

\section{DISCUSSÃO}

Desde o começo do estudo, durante a coleta de dados, já foi possível vislumbrar o quanto $\mathrm{o}$ ato de cuidar exige das pessoas. Apesar de participarem de grupos de apoio, 26 pessoas não se dispuseram a conversar sobre suas tarefas como cuidadores. A maior proporção de aposentadas no estudo sugere a disponibilidade de tempo como variável interveniente das interpretações que podemos tirar deste estudo. Além disso, aqueles que estavam começando a frequentar o grupo de apoio poderiam se encontrar mais fragilizados com o diagnóstico recente da demência do familiar e com maior dificuldade para fazer parte da pesquisa.

Neste estudo, houve o predomínio de filhas adultas e com alta escolaridade. É unânime, na literatura nacional e internacional, a presença da mulher como cuidadora, geralmente esposa e filha. ${ }^{5,12,14,19-23}$ As mulheres, antigamente, não desempenhavam funções fora de casa, o que lhes dava maior disponibilidade para o cuidado da família e da casa. Alguns autores também encontraram a filha como principal cuidadora familiar nas suas pesquisas. ${ }^{9,20,24} \mathrm{Na}$ vida familiar, existe uma hierarquia de rede de apoio: em primeiro lugar, vem a esposa e, em seguida, vêm os descendentes diretos, sendo a filha mais velha a candidata mais provável. Raramente o cuidador é uma pessoa do gênero masculino, outro parente ou uma pessoa jovem, apesar de que, hoje, o homem tem se revelado nesse processo. ${ }^{8}$ Vale acrescentar que a sobrevida da mulher no Brasil é de mais de oito anos em relação ao homem. ${ }^{1}$

Encontramos maior percentual de filhas do que esposas, provavelmente por se tratar da população que participa de grupos de apoio. Em estudo desenvolvido por Karsh ${ }^{5}$ no município de São Paulo, em 102 domicílios de pessoas com mais de 50 anos pós-acidente vascular cerebral, observou-se que $92,9 \%$ dos cuidadores entrevistados eram do sexo feminino, a maior parte era formada por esposas (44,1\%), seguida por filhas (31,3\%). Karsh encontrou $59 \%$ das cuidadoras com idade acima de 50 anos e $41 \%$ com mais de 60 anos. Além disso, 39,3\% das cuidadoras tinham entre 60 e 80 anos e cuidavam de $62,5 \%$ de pacientes da mesma faixa etária, o que mostra que pessoas idosas estão cuidando de idosos. Entretanto, outros estudos, assim como este, mostraram cuidadoras de idade mais jovem, provavelmente porque o ato de cuidar exige uma pessoa com boa condição de saúde. ${ }^{12,20,22,24}$

A participação em grupos de apoio pode ter selecionado uma população de maior escolaridade, como observado em alguns trabalhos. $^{12,20}$ Em estudos realizados em comunidade ou com cuidadores do Programa de Saúde da Família (PSF), a realidade é outra, com a maior parte dos cuidadores apresentando grau de instrução até o ensino fundamental., ${ }^{6,21,24}$ Em relação às cuidadoras que possuíam nível superior, essa situação pode favorecer o cuidado prestado, pelo acesso às informações, sobretudo em relação à administração de medicamentos.

As cuidadoras, neste estudo, dormiam menos de oito horas por noite. Apesar de a necessidade de sono ser muito individual, distúrbios do sono são comuns entre cuidadores de pessoas com demência. Estimativas sugerem que existem mais de dez milhões de cuidadores, sendo que dois terços deles experimentam alguma forma 
de distúrbio do sono durante todo o processo de cuidar. ${ }^{25} \mathrm{Um}$ conjunto de fatores predisponentes e perpetuantes, como a presença do sono interrompido, sobrecarga, depressão do cuidador e saúde física comprometida, está associado às queixas de sono. ${ }^{25} \mathrm{Em}$ estudo com os cuidadores familiares de pacientes dependentes no PSF, mais da metade dormia em média sete horas. ${ }^{19}$

A prevalência das doenças autorreferidas, principalmente dores nas costas e articulares, colesterol alto e hipertensão arterial, e do uso de medicamentos para depressão e hipertensão, foi observada nesta pesquisa. Algumas doenças provocam uma série de sinais e sintomas que podem interferir na avaliação do cuidador sobre sua vida e saúde. ${ }^{19}$ Algumas cuidadoras justificam suas dificuldades do ato de cuidar pelo próprio envelhecimento ou por sua condição de saúde, com sintomas físicos e/ ou psíquicos. ${ }^{21}$ Estudos nacionais relatam o predomínio do diagnóstico de hipertensão e do uso crônico de medicamentos, inclusive antidepressivos, hipnóticos e ansiolíticos, além de algum tipo de dor ou doença. ${ }^{19,21}$ Estudo sobre o suporte domiciliar aos adultos com perda de independência e o perfil do cuidador observou maior prevalência de dores lombares $(40,7 \%)$, depressão (39,0\%), artrite e reumatismo $(37,3 \%)$, problemas cardíacos $(10,2 \%)$ e diabetes $(5,1 \%) .{ }^{26}$

Em relação aos serviços de saúde, foi observado que 59 cuidadores procuraram pelos atendimentos de saúde, o que foi um dado positivo, pois os familiares tendem a deixar de lado a própria saúde para cuidar do paciente. Poucos relatam qualquer tipo de doença, mas todos expressam cansaço, estresse e necessidade de algum tempo para si mesmos. ${ }^{22} \mathrm{Em}$ contrapartida, Garrido $\&$ Menezes $^{12}$ observaram em um estudo que, clinicamente, a maioria dos cuidadores apresentou saúde física excelente ou boa.

A Escala de Sobrecarga de Zarit utilizada obteve alpha de Cronbach de 0,72 , representando um índice de valor moderado referente à consistência interna da escala para essa amostra, e inferior ao valor apresentado em estudos maiores com populações de cuidadores de idosos com depressão e participantes do PSF por Scazufca ${ }^{14}$ e Amêndola,${ }^{19}$ com valores do alpha de Cronbach de 0,87 e 0,86 , respectivamente.

Considerando as análises das perguntas inseridas na Escala de Sobrecarga de Zarit, seguidas de suas frequências, foi possível observar que, quanto às respostas "nunca se sentem sobrecarregados", encontrou-se uma frequência maior de respostas $(>50 \%$ na tabela) dos cuidadores em relação a sentir-se envergonhado com o comportamento do idoso, sentir-se irritado quando está junto com seu familiar, não sentir vontade de receber visitas em casa, achar que não vai ser capaz de cuidar do seu familiar por muito tempo, e não querer entregar o cuidado do seu familiar a outra pessoa. Já em relação a uma maior sobrecarga ( $>30 \%$ na tabela), quando os cuidadores responderam que "quase sempre se sentem sobrecarregados", observou-se uma relação com situações como: sentir que o idoso depende do cuidador, sentir receio pelo futuro que aguarda o familiar, não dispor de dinheiro suficiente para cuidar do familiar e ser a única pessoa com quem o familiar pode contar. Em relação à frequência com que "se sentiram sobrecarregados ao ter que cuidar do familiar", muitos cuidadores $(48,3 \%)$ responderam "às vezes".

Luzardo et al. ${ }^{2}$ observaram, em seu estudo, que em relação às respostas "nunca se sentem sobrecarregados", $58,3 \%$ dos cuidadores relataram que seus familiares nunca pediam ajuda além do necessário, $80,6 \%$ dos cuidadores relataram nunca se sentirem envergonhados, $61,1 \%$ ficavam irritados ou tensos quando o familiar estava por perto, e $61 \%$ sentiam que o idoso não afetava negativamente seus relacionamentos com outros membros da família ou amigos. Adicionalmente, $63,9 \%$ dos cuidadores relataram que nunca se sentiam pouco à vontade para receber visitas em casa, 47,2\% afirmaram que nunca sentiram que não tinham privacidade com o seu familiar dementado, e 5,6\% dos cuidadores nunca se sentiram incapazes de cuidar do seu familiar por muito mais tempo. Em relação à resposta 
"sempre se sente sobrecarregado", 75\% dos cuidadores relataram que seus familiares sempre dependiam deles, e que sentiam que o idoso esperava ser sempre cuidado por eles, como se o cuidador fosse a única pessoa com que ele pudesse contar. Quando questionados sobre a frequência com que, "de maneira geral, se sentiram sobrecarregados por cuidar do dementado", os cuidadores dividiram-se entre os extremos da escala: $21(42,8 \%)$ responderam "nunca ou raramente" e 19 (38,7\%), "frequentemente ou sempre".

Este estudo não encontrou relatos de sobrecarga severa, apenas leve e moderada, provavelmente porque pessoas muito sobrecarregadas não participam de grupos de apoio. ${ }^{27}$ Esses valores foram observados em categorias de sobrecarga associadas às características dos cuidadores, em estudos de Amendola et al. ${ }^{19}$ e Luzardo et al. ${ }^{2}$ com 66 cuidadores participantes do PSF e com 36 em um serviço de neurogeriatria, respectivamente.

A chance de as cuidadoras aposentadas terem sobrecarga moderada é maior do que nas cuidadoras que trabalhavam fora, pois, apesar da idade, elas são consideradas pessoas mais disponíveis, estando mais "aptas" para cuidar do familiar dementado, que geralmente é o cônjuge ou um dos pais. Além da maior idade, a sobrecarga tende a se tornar maior diante do despreparo para desempenhar novos papéis e da falta de informação sobre como entender e enfrentar a doença, e também pela falta de ajuda de outras pessoas, pelas condições financeiras e pela execução das tarefas numa fase em que os aposentados estão querendo desfrutar mais da vida. Por outro lado, em um estudo, o ato de cuidar é considerado por algumas cuidadoras como uma "missão", "destino", algo que deve ser desempenhado individualmente, então elas acabam não compartilhando os cuidados com outros familiares, o que gera a sobrecarga. ${ }^{6}$

Houve associação significativa entre a prática de esportes e a sobrecarga, em que aqueles que praticavam mais esporte apresentavam maior sobrecarga, dado contrário ao da literatura, pois é sabido que o lazer e a prática de esportes são atividades que contribuem para nossa saúde física e mental. ${ }^{28}$ Devido ao desenho transversal, podemos observar o efeito de causalidade reversa, ou seja, por se tratar de uma população com alta escolaridade e acesso, as cuidadoras com maior sobrecarga foram as que procuraram, no esporte, uma válvula de escape para suas atividades de cuidar.

As pessoas que apresentaram sobrecarga moderada podem ter a religião como uma forma de enfrentamento, a fim de dar conta dessa nova fase de vida como cuidadoras. A fé pode ser um atributo necessário, que proporciona apoio e alívio nas tensões, encorajando, dando apoio e esperanças. Artigos internacionais consideram importante o suporte familiar e a religiosidade para diminuir o estresse e os sintomas de depressão dos cuidadores, e melhorar a autoestima, o autocuidado e a consequente preservação da saúde mental dos mesmos. ${ }^{29,30} \mathrm{Um}$ estudo verificou que os cuidadores aumentaram sua participação em atividades religiosas para se aproximarem de outras pessoas com valores semelhantes e aceitarem o cotidiano e os sentimentos que afloram. ${ }^{31}$ Esses sentimentos podem ser ambíguos, do tipo positivo, como amor, consideração e carinho, e/ou negativo, como insegurança, raiva, culpa, solidão, medo e compaixão.

Quanto à tarefa de cuidar, foi possível observar que as participantes da pesquisa com tendência a um maior tempo na função de cuidadora ( $\geq 60$ meses) apresentaram mais chance de ter sobrecarga moderada. Em estudo com 25 octogenários e seus respectivos cuidadores sobre a qualidade de vida, o tempo médio como cuidador foi de dois anos e oito meses. ${ }^{20}$ Cassis et al., ${ }^{32} \mathrm{em}$ seu estudo, observaram que o tempo de cuidado teve uma média de dois anos e três meses, e que o tempo de evolução da doença foi de dois anos e meio, sugerindo que o aumento no impacto emocional é proporcional ao tempo de evolução da doença e ao tempo de convívio. ${ }^{32}$

A não associação da sobrecarga ao grau de dependência do idoso, a transtornos mentais 
comuns e a queixas de memória nos leva a refletir que, apesar de os idosos serem altamente dependentes, os cuidadores tinham apoio de outros familiares; cuidavam, na medida do possível, da saúde; faziam uso de medicamentos; e também frequentavam os grupos de apoio. Os transtornos mentais foram frequentemente relatados, assim como o uso de ansiolíticos e antidepressivos. A escala utilizada (SRQ) revelou um bom grau de fidelidade, apesar de menor que o encontrado em outro estudo ( $\alpha=$ $0,62 \times \alpha=0,80,)^{16}$

As associações relevantes foram dores nas costas, esporte e tempo de cuidado diário. Aquelas que apresentaram dores nas costas tiveram mais chance de ter transtornos mentais comuns. De acordo com Goldenberg, ${ }^{33}$ a dor nas costas está diretamente relacionada a uma série de fatores, como: idade, sexo, tabagismo, erros posturais do dia a dia, sedentarismo, obesidade, hereditariedade e, sobretudo, depressão. Se mais de $80 \%$ da população brasileira sofre de dores nas costas, podemos dizer que mais de $70 \%$ das pessoas com quadro de depressão têm o mesmo problema.

A prática de esporte apresentou associação com os transtornos mentais comuns, em que aqueles que não praticavam esportes apresentaram mais chance de ter transtornos mentais comuns, o que é esperado, pois pessoas que praticam esporte tendem a apresentar menos problemas emocionais. Matsudo \& Matsudo $^{34}$ apontam como aspectos positivos da atividade física: a melhora do autoconceito, da autoestima e da imagem corporal; a diminuição do estresse e da ansiedade; a melhora da tensão muscular e do sono; a diminuição do consumo de medicamentos; e a melhora das funções cognitivas e da socialização. Além disso, o exercício físico também induz a liberação de neurotransmissores, como a serotonina, noradrenalina e a dopamina, que auxiliam na redução do estresse, ansiedade e depressão. ${ }^{28}$

As cuidadoras que cuidavam parcialmente $(<24$ horas) apresentaram maior chance de ter transtornos mentais comuns. Em estudo que buscou avaliar o impacto subjetivo da doença de
Alzheimer na vida de 29 cuidadores primários, observou-se que a maioria afirmou dedicar de 19 a 24 horas por dia aos cuidados. ${ }^{22}$ Em outra pesquisa qualitativa com 14 familiares, dez cuidadores afirmaram que a carga horária diária era acima de 12 horas, e que alguns membros da família se sentem abatidos pela demanda nas 24 horas de cuidados diários, podendo levar até ao rompimento de suas atividades como cuidadores. ${ }^{35}$

Também se observou grande frequência de queixas de memória, com apenas uma associação significativa ao uso de antidepressivos. O instrumento de aferição da memória apresentou boa confiabilidade interna $(\alpha=0,74)$, semelhante à literatura. ${ }^{10} \mathrm{O}$ uso de medicamentos de ação central, como os anti-hipertensivos e os antidepressivos, pode causar sintomas depressivos e queixas de memória, devido à dificuldade de atenção. ${ }^{28} \mathrm{Um}$ estudo relatou que as queixas de memória estão associadas à memória em idosos e, principalmente, em adultos jovens, devido à depressão e à ansiedade. ${ }^{11}$

Este é um estudo transversal, que observou o grau de sobrecarga e as variáveis associadas em um mesmo momento no tempo, sem poder para determinar causalidade. A população de estudo, cuidadoras participantes de grupos de apoio, não representa o universo de cuidadores de pacientes com demência. Além disso, o pequeno tamanho da amostra não permite a generalização de seus resultados. Apesar dessas limitações, o presente estudo tentou trazer alguma contribuição sobre como cuidadores percebem a sobrecarga, os transtornos emocionais e as queixas de memória, como incentivo a pesquisas futuras sobre o tema.

\section{CONCLUSÕES}

O estudo de cuidadoras que frequentam grupos de apoio mostrou maior participação de filhas adultas e de alta escolaridade, com menores graus de sobrecarga, mas com presença de comorbidades, uso de medicamentos, grande frequência de transtornos mentais comuns e queixas de memória. 
O fato de as cuidadoras terem uma vida ativa e não serem cuidadoras únicas, assim como participarem de grupos de apoio, pode ter contribuído para minimizar essa sobrecarga. Essa participação deve ser incentivada, pois os cuidadores têm no grupo de apoio um suporte social, no sentido de aprender mais sobre a doença e como conviver melhor com o familiar, de compartilhar sentimentos e experiências e de conscientizar-se da importância de se cuidar para cuidar bem do outro, adaptando-se melhor à função de cuidador.

\section{REFERÊNCIAS}

1. Instituto Brasileiro de Geografia e Estatística. Censo demográfico,2010. Rio de Janeiro http://www.ibge. gov.br

2. Luzardo AR, Gorini MIPC, Silva APSS. Características de idosos com doença de Alzheimer e seus cuidadores: uma série de casos em um serviço de neurogeriatria. Texto Contexto Enferm. 2006 out-dez;15(4):587-594.

3. Lima-Costa MF, Guerra HL, Barreto MS, Guimarães RM. Diagnóstico de saúde da população idosa brasileira: um estudo da mortalidade e das internações hospitalares públicas. Informe Epidemiológico do SUS 2000 jan-mar; 9(1):23-41.

4. Resende MCF, Dias EC. Cuidadores de Idosos: um novo/velho trabalho Physis Revista de Saúde Coletiva, 2008;18(4):785-800.

5. Karch UM. Idosos dependentes: famílias e cuidadores. Cad. Saúde Pública. Rio de Janeiro, 2003 mai-jun;19(3): p.861-66.

6. Caldeira APS, Ribeiro RCHM. O enfrentamento do cuidador do idoso com Alzheimer. Arq. Ciênc Saúde 2004 abr-jun;11(2):2-6.

7. O'Brien J. Caring for caregivers.American Academy of Family Psysicians, 2000 December Disponível em URL:<http://www.aafp.org/afp/AFP printer/20001215/editorials.html>

8. Cattani RB, Girardon-Perlini NMO. Cuidar do idoso doente no domicílio na voz de cuidadores familiares. Revista Eletrônica de Enfermagem: 2004.6(2) : p.254271. Disponível em URL: <http:// www.fen.ufg.br>

9. Aguglia E, Onor ML, Trevisiol M, Negro C, Saina M, Maso E. Stress in the caregivers of Alzheimer's patients: an experimental investigation in Italy.

Diante disso, faz-se necessário o apoio a esses indivíduos, por meio de programas de atendimento domiciliar, serviços de cuidador substituto para aliviar a sobrecarga, mais informações, orientações, encaminhamento e apoio dos profissionais da área da saúde.

Considerando os quadros ansiosos e depressivos que comprometem a cognição, tais queixas de memória merecem atenção, pois sugerem que esses sintomas devem ser investigados e tratados.

American Journal of Alzheimer's Disease and Other Dementias. 2004 July-aug; v.19 (4):248-52.

10. Benites D. Auto-eficácia geral e auto-relato de falhas de memória prospectiva e retrospectiva em adultos e idosos. [Dissertação] Universidade Federal do Rio Grande do Sul, RS, 2006.

11. Paulo DLV, Yassuda MS. Queixas de memória de idosos e sua relação com escolaridade, desempenho cognitivo e sintomas de depressão e ansiedade. Rev. Psiq.Clin. 2010; 37(1): 23-6.

12. Garrido R, Menezes PR.Impacto em cuidadores de idosos com demência atendidosem um serviçopsicogeriátrico.Rev.SaúdePública 2004;38(6):835-841.

13. Palma KAXA.Efeitos do envelhecimento e do estresse crônico sobre a memória declarativa. [Tese]. PUCRS Porto Alegre (RS);2004.

14. Scazufca M Brazilian version of the Burden Interview scale for the assessment of burden of care in carers of people with mental illness. Rev Bras Psiquiatr, 2002:24(1):12-7.

15. Mari JJ, Williams P. A validity study of a psychiatric screening questionnaire (SRQ-20) in primary care in the city of São Paulo. British Journal of Psychiatry, 1986; 148: p. 23-6.

16. Santos KOB, Araújo TM, Oliveira NF. Estrutura fatorial e consistência interna do Self-Reporting Questionnaire (SRQ-20) em população urbana. Cad.. Saúde Pública,2009 jan; 25(1):214-222.

17. Aquino et al Estudo Longitudinal de Saúde do Adulto, ELSA BRASIL 2011. DisponívelemURL: $<$ http://bvsms.saude.gov.br/bvs/ publicações/decit_10anos.pdf $>$ 
18. Pfeffer RI, Kurosaki TT, Harrah Jr. MA, Chance JM, Filos S. Measurement of functional activities in older adults in the community. Journal of Gerontology. 1982; 37(3):p.323-9.

19. Amendola F, Oliveira MAC, Alvarenga MRM. Qualidade de vida dos cuidadores de pacientes dependentes no programa de saúde da família , 2008 Abr-Jun; 17(2):266-72.

20. Inouye K, Pedrazzani ES, Pavarini SCI. Octogenários e cuidadores: perfil sócio-demográfico e correlação da variável qualidade de vida. Texto contexto enferm, 2008, abr-jun;17(2):350-7.

21. Giacomin KC, Uchoa E, Firmo JOA, LimaCosta MF. Projeto Bambuí: um estudo de base populacional da prevalência e dos fenômenos associados à necessidade de cuidador entre idosos. Cad. Saúde Pública 2005 jan-fev;.21(1):80-91.

22. Lemos ND, Gazzola JM, Ramos LR. Cuidando do paciente com Alzheimer:o impacto da doença no cuidador. Saúde e Sociedade 2006 set-dez ;15(3), p.170-9.

23. Etters L, Goodall, D, Harrison BE. Caregiver burden among dementia patient caregivers: a review of the literature. Journal of the American Academy of Nurse Pratitioners. 2008: 423-8.

24. Nakatani AYK, Souto CCS, Paulette L M, Melo TS, Souza MM. Perfil dos cuidadores informais de idosos com déficit de autocuidado atendidos pelo Programa de Saúde da Família. Revista Eletrônica de Enfermagem 2003; 5(1):15-20. Disponível em URL:<http:www. fen.ufg.br/revista>

25. McCurry SM, Logsdon RG, Teri L, Vitiello MV. Sleep disturbances in caregivers of persons with dementia: contributing factors and treatment implications. Sleep Med Rev.2007, April; 11(2): 143-153.

26. 26.Karsh UMS (org.). Envelhecendo com dependência: revelando cuidadores. São Paulo: EDUC;1998.
27. Seima, MD, Lenardt, MH. A sobrecarga do cuidador familiar de idoso com Alzheimer Textos \& Contextos (Porto Alegre), Ago/Dez 2011, 10(2): p.388- 398.

28. Stella F, Gobbi S, Corazza DI,Costa JLR . Depressão no idoso: diagnóstico, tratamento e benefícios da atividade física. Motriz,2002, ago/dez; 8(3): pp.91-9.

29. Zunzunnegui M-V Béland F,Llácer A, Keller I. Family, religion, and depressive symptoms in caregivers of disabled elderly. J Epidemiology Community Health, 1999; 53:p 364-9.

30. Murray-Swank AB, Lucksted, A, Medoff DR,Yang Y,Wohlheiter K, Dixon LB. Religiosity, psychosocial adjustment and subjective burden of persons who care for those with mental illness. Psychiatric services, 2006 march; 57(3):361-365

31. Travensolo CF, Karsh UMS.Qualidade de vida de um grupo de cuidadores familiares de portadores de doença de Alzheimer. Revista Kairós. 2004 dez.; 7(2): p.241-258.

32. Cassis SVA, Karnakis T,Moraes TA, Curiati JAE, Quadrante ACR, Magaldi RM. Correlação entre o estresse do cuidador e as características clínicas do paciente portador de demência. Rev. Assoc. Médica Bras 2007.53(6):497-501

33. Goldenberg E. Dor nas costas: uma auto-avaliação pode ajudar muito [citadoem10/01/2006] DisponivelemURL:<http://wmw.hospitalar.com/opiniao/ opiniao1180.html>.

34. Matsudo SM, Matsudo VKR, Neto TLB. Efeitos benéficos da atividade física na aptidão física e saúde mental durante o processo de envelhecimento. Revista Brasileira de Atividade Física e Saúde, 2000;5(2):p.60-76.

35. Santos SSC, Pelzer MT, Rodrigues MCTR. Condições de enfrentamento dos familiares de cuidadores idosos portadores de doença de Alzheimer, RBCEH, 2007 jul-dez v.4(2):p.114-126. 